\title{
Radar Surveillance in Urban Environments
}

\author{
Dave Tahmoush, Jerry Silvious, and Bob Bender \\ US Army Research Laboratory \\ 2800 Powder Mill Rd \\ Adelphi, MD
}

\begin{abstract}
Radar surveillance in difficult environments like urban areas can be challenging due to large amounts of both multipath and clutter. Additionally, buildings and clutter like parked vehicles can produce shadowed areas where the line-ofsight is broken. We analyzed urban materials to determine how to utilize multipath to see into the shadows of urban environments, which polarization has the least loss, and which frequencies performed the best across a range of environments. Urban canyons were analyzed to determine whether there was more of a multipath effect on the measurements or a waveguide effect. The detection and tracking of non-line-of-sight moving objects using multiple bounces was attempted across a variety of urban building materials with an urban radar surveillance system. We demonstrate the detection and tracking of subjects using multipath returns, but there was no disambiguation of real or multipath sources. We also discuss the challenges of classification in an urban environment.
\end{abstract}

\section{INTRODUCTION}

Information collection with surveillance sensors is a valuable ability in urban environments. A sensor with capacity to identify moving objects even when they are around a corner, both indoors and outdoors, is an asset in an urban engagement. Even though urban environments are complicated by back-scattering and multiple-bounce pathways contributing to the received radar signals, radars are becoming increasingly important for security-related reconnaissance and surveillance tasks. Currently surveillance in urban areas is dominated by optical devices. Sensitive areas inside a city are covered by a large network of cameras. Optical images are the best means of surveillance for an operator because they do not need interpretation. However, they work only in line-of-sight (LOS). In order to prevent blind areas, it is necessary to employ a dense network of cameras. Additionally, their performance degrades in conditions such as smoke and low light. Additionally, cameras in public areas bring up issues of privacy. Many of these issues are mitigated through the use of radar.

In an urban environment there are multiple challenges for radar surveillance, such as a lack of direct observations, obscuration from buildings, and the clutter returns from buildings and other objects $[1,2,3]$. In order for the radar to perform well in the complicated urban environment and achieve high performance accuracies, the surveillance system must be capable of incorporating urban environment features and adapt dynamically to its changes. For example, using optical urban imagery, building obscuration and reflections can be predicted and then target localization can be improved. Also, multipath can be used as an indirect means to view a target which is not in direct line-of-sight (LOS).

Multipath returns can be treated as interference and mitigated [4, 5]. However, there are scenarios in the urban environment where the direct path is lost, and the only paths available utilize multipath. Utilizing prior knowledge about the environment like imagery, road maps, and building locations, the multipath returns can be used to confirm the target detection [6]. Multipath propagation can be utilized [7], and target information can be extracted from multipath returns to enhance tracking performance. Especially when no LOS is available, multipath returns allow the detection and tracking of a target without LOS, decreasing the obscuration effects from buildings and increasing the visible area in the urban environment [1]. The standard data association techniques, such as the nearest neighbor standard filter, optimal Bayesian data association filter [8] or the probabilistic data association filter $[9,10]$, have to be customized to accommodate the multipath returns. One multipath data association problem is faced in over-the-horizon radar [11], but only for the initiation and tracking of non-maneuvering targets with constant probability of detection. A multipath data association technique can be used to find the LOS measurement, mitigating the non-LOS measurements [12].

Utilizing ground moving target indicator (GMTI) radar in an urban environment requires a functional understanding of the multipath environment. Measurements in a complex urban environment create multiple returns for each target based upon the number of reflections. In addition to a high number of material classes and surface properties, transmission and reflection properties from walls are a function of both reflection angle and frequency. Here we consider the reflection properties of various wall materials and report multipath radar measurements in various urban canyon environments.

\section{URBAN REFLECTION MEASUREMENTS}

In order to maximize the performance of an urban radar surveillance application, we measured the reflection properties of a number of urban materials to try to optimize both the frequency and the polarization of the radar system. Data was 
collected with ARL's ISAR imaging system to determine the best frequency and polarization for urban operations. This system is a fully polarimetric coherent pulsed radar that operates in $\mathrm{X}, \mathrm{Ku}, \mathrm{Ka}$, and $\mathrm{W}$-bands [13], so it gives a broad and consistent measurement at a number of frequencies and polarizations.

Wall sections were constructed for the measurements on top of a steel turntable which was partially covered by absorber to mitigate its reflection. A resolver inside of the turntable provides precise position feedback to the data collection system. There was one wall section made of each of the following materials: plywood, stucco, brick, and concrete block. The wall setup is in Figure 1, while a diagram of the measurement setup is in Figure 2. Fully polarimetric coherent data was collected for each frequency. The data was calibrated to correct for I and Q imbalance and polarization distortion [13]. The data was also calibrated against a known calibration reflector to provide the Radar Cross Section (RCS) of a trihedral in the scene. The data collected included RF energy reflected from the wall, turntable, and ground as well as the trihedral of interest. To discern the trihedral's reflected energy from the rest of the reflected energy, RCS images were generated for each of the measurements. The area of the image corresponding to the trihedral was then masked off and all of the energy in this part of the image was summed together to generate the trihedral's reflected RCS. The measured two-way attenuation variation with frequency and wall type is in Table 1 for $\mathrm{VV}$ and $\mathrm{HH}$ polarizations. The two-way attenuation is relevant because the signal is going to be reflected by the same material for most non-LOS measurements.

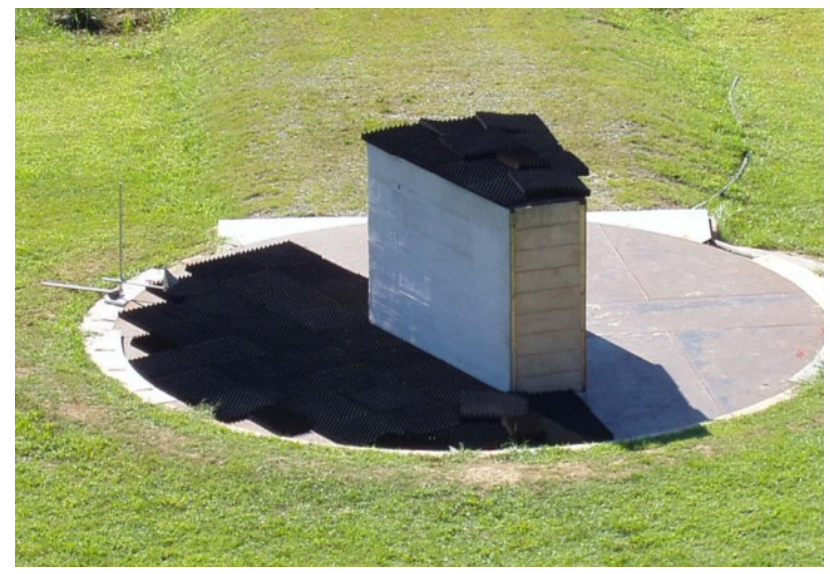

Figure 1. The wall as prepared for measurement.

The experiments showed high variability with respect to both frequency and angle, with characteristics seen due to surface roughness and multiple reflections through more transmissive materials that could be separated in the analysis. The response varied significantly with polarization, and could be approximated by a flat surface response with an additional grating term for patterned surface roughness. For utilizing multipath to see into the shadows of urban environments, VV polarization often has the least loss. Additionally, the X-band measurements often had lower loss than the $\mathrm{Ku}$ or Ka-band measurements, as can be seen in Table 1. Therefore we used a mobile X-band VV system to make additional measurements in urban settings.

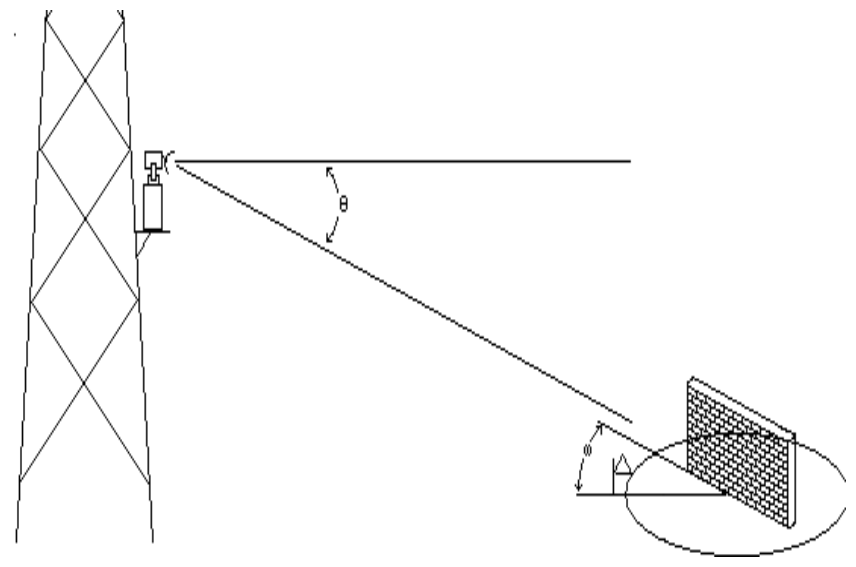

Figure 2. Diagram of the measurement setup.

TABLE I. TWO-WAY ATtENUATION VARIATION

\begin{tabular}{|l|l|l|l|}
\hline & X-Band & Ku-Band & Ka-Band \\
\hline Wood VV & $10-23 \mathrm{~dB}$ & $8-19 \mathrm{~dB}$ & $13-38 \mathrm{~dB}$ \\
\hline Stucco VV & $5-17 \mathrm{~dB}$ & $6-18 \mathrm{~dB}$ & $10-25 \mathrm{~dB}$ \\
\hline Block VV & $10-22 \mathrm{~dB}$ & $5-20 \mathrm{~dB}$ & $5-20 \mathrm{~dB}$ \\
\hline Brick VV & $5-18 \mathrm{~dB}$ & $7-24 \mathrm{~dB}$ & $10-25 \mathrm{~dB}$ \\
\hline Wood HH & $15-33 \mathrm{~dB}$ & $15-40 \mathrm{~dB}$ & $20-60 \mathrm{~dB}$ \\
\hline Stucco HH & $3-17 \mathrm{~dB}$ & $15-30 \mathrm{~dB}$ & $17-50 \mathrm{~dB}$ \\
\hline Block HH & $10-35 \mathrm{~dB}$ & $11-43 \mathrm{~dB}$ & $12-57 \mathrm{~dB}$ \\
\hline Brick HH & $12-35 \mathrm{~dB}$ & $16-45 \mathrm{~dB}$ & $20-54 \mathrm{~dB}$ \\
\hline
\end{tabular}

\section{DOPPLER RESPONSE IN A MULTIPATH ENVIRONMENT}

The equation for computing the non-relativistic Doppler frequency shift, $F_{d}$, of a simple point scatterer moving with speed $v$ with respect to a stationary transmitter is:

$$
F_{d}=F_{t} \frac{2 v}{c} \cos \theta \cos \phi
$$

where $F_{t}$ is the frequency of the transmitted signal, $\theta$ is the angle between the subject motion and the beam of the radar in the ground plane, $\phi$ is the elevation angle between the subject and the radar beam, and $c$ is the speed of light. For complex objects, such as walking humans, the velocity of each body part varies over time. Additionally, the radar cross-section of various body parts is a function of aspect angle and frequency.

The typical Doppler response of a point target in a multipath environment changes due to the different paths that the transmitted and reflected signal can take. A diagram of possible paths in an urban canyon is shown in Figure 3.

The Doppler response of a point target in a multipath environment becomes a function of range for the different lengths of the possible paths. At one particular range and for one particular set of incoming and outgoing paths, the Doppler is:

$$
F_{d}=F_{t} \frac{v}{c}\left(\cos \theta_{i} \cos \phi_{i}+\cos \theta_{o} \cos \phi_{c}\right)
$$

where $\theta_{1}$ and $\phi_{\mathrm{i}}$ are the angles of the incoming wave and $\theta_{\mathrm{o}}$ and $\phi_{\mathrm{o}}$ are the angles of the outgoing wave. There are 
multiple paths that can exist to the target at the same range, and these combine coherently. When the model has range extent and is not merely a point source, these interactions become more complex. Additionally, the particular radar will have a range resolution that will combine multiple paths together.

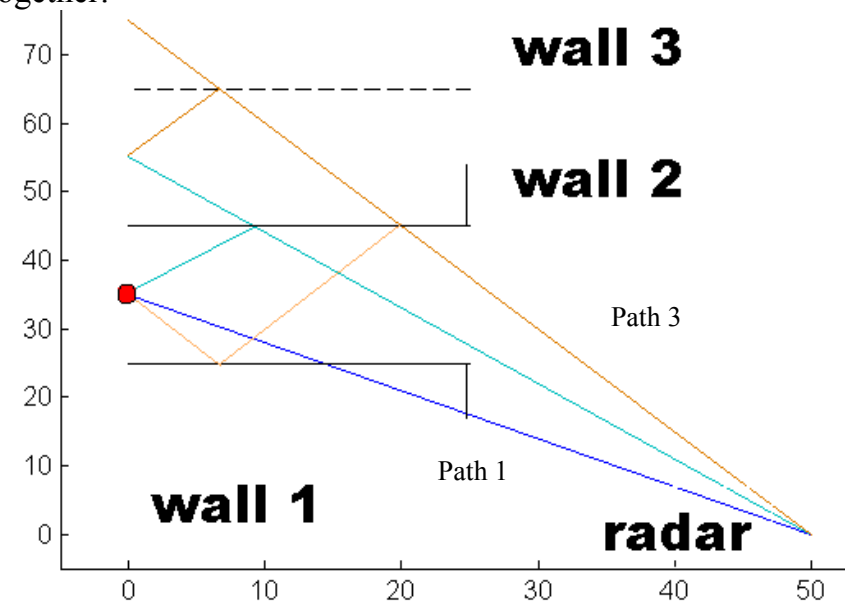

Figure 3. Possible paths within an urban canyon to a target. In this case, the direct path is blocked by wall 1 . Wall 3 is a phantom wall, used to project the reflections from wall 1 after reflection from wall 2. For a perfect radar and a point source, the multiple reflections can look like multiple targets at ranges that would sometimes place them inside buildings.

The response of a single target in a complex environment can be modeled as multiple targets in a simpler environment. This is shown in Figure 4, where three possible paths are mapped out for incoming or outgoing waves. Because of the different angles with respect to the motion of the target, the Doppler response will vary depending on the paths that the radar energy takes. A simulated range-Doppler chip is in Figure 5, using the geometry of Figure 3 and a point reflector moving at $2 \mathrm{~m} / \mathrm{s}$ along the urban canyon.
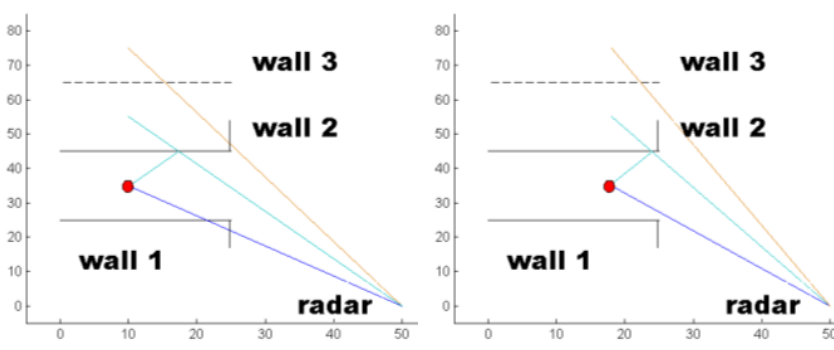

Figure 4. Examples of point targets moving in an urban canyon. On the left, the direct path is blocked by wall 1 , while wall 2 reflects one indirect path. On the right, the direct path is finally available, and one indirect path is still available.

The Doppler response of a point target in a multipath environment can be considered as the summation over all possible paths combined coherently for a given geometry. For the urban canyon setup in Figure 3, the direct path does not give a response, but several other paths should. Considering that each reflection will give a loss as shown in Table 1 that will be incorporated into the returned energy, and further simplifying to a ground scenario where $\phi_{\mathrm{i}}=\phi_{\mathrm{o}}=0$, the set of Doppler responses from a point target will be:

$$
F_{d}(r=r 1+r 2)=\sum_{\substack{\text { incoming } \\
\text { path } \\
r 1}} \sum_{\begin{array}{c}
\text { outgoing } \\
\text { path } \\
r 2
\end{array}} F_{t} \frac{v}{c}\left(\cos \theta_{i}+\cos \theta_{o}\right)
$$

where $r=r 1+r 2$. Note that as you include realistic target sizes and radar range resolutions, the phases will need to be included to combine the signals coherently. The different paths produce various angles reflected off of the walls, and more attenuation due to more reflections. The result of motion along the canyon is a "tail" of reflected signal with reducing frequency and amplitude extending in range from the target. A tail can be discrete trackable returns or a continuum of energy extending out in range depending on the extent of the subject and the resolution of the radar. A simulated tail is in Figure 5, and examples of measured tails can be seen in Figure 6 .

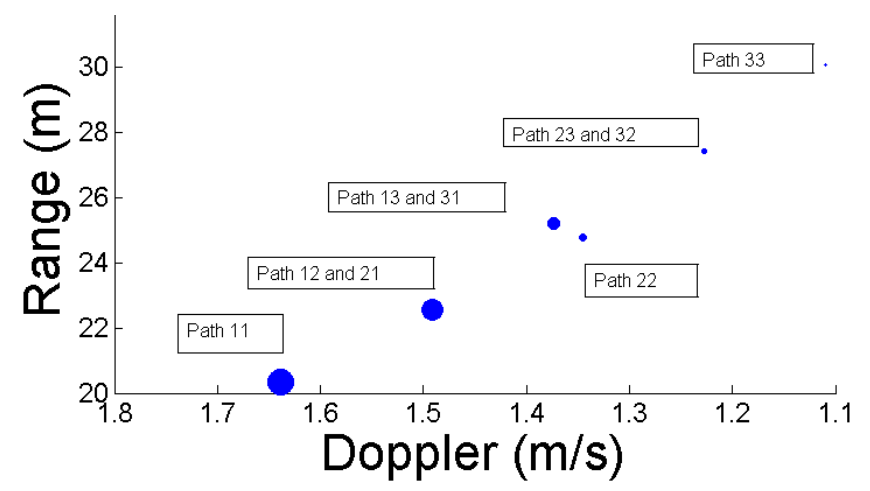

Figure 5. Range-Doppler response of a point target moving in an urban canyon. The size corresponds to the amplitude with attenuation due to reflections, while the Doppler and range assume the geometry from Figure 3.

The particular geometry of an urban canyon scenario has a significant effect on the resulting ability to track. RangeDoppler chips of the person in motion along the urban canyon are shown in Figure 7, where multiple chips have been removed when there are no detections. The direct and multipath response from a dismount walking between two metal and glass buildings is in Figure 8. The walker was close to the near building, which means that there were times when the shadow of the near building blocked the direct path and there was no indirect path. This shows up as gaps in the range-time image. This also means that the probable tail will be almost continuous, since the distance to the next reflection at the near building is small, and two of these tails are expanded in Figure 6.

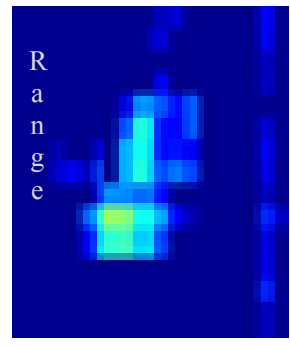

Doppler

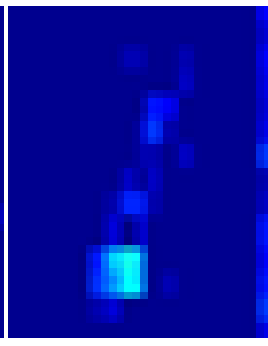

Doppler

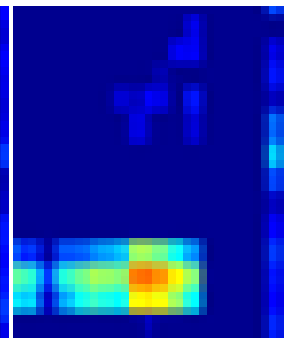

Doppler
Figure 6. Examples of tailed targets moving in an urban canyon compared to a normal target (right) with direct response. 

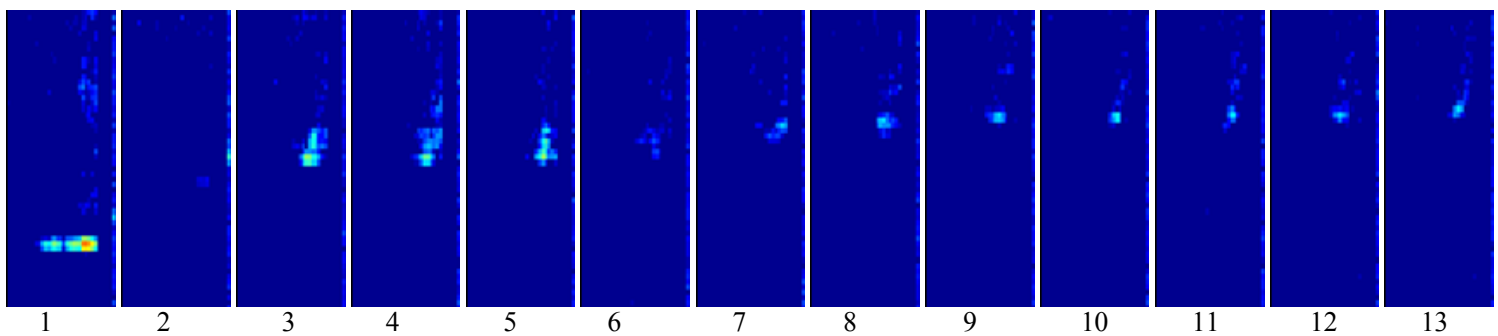

Figure 7. Range-Doppler chips of a person moving out from between two brick and glass buildings. The direct response is in chip 1, where there is significant micro-Doppler, and there is a significant amount of time where there is minimal response like chip 2, and then the reflected response in chips 3 through 13. This corresponds to the activity in Figure 8. The indirect path has a reduced return strength, which makes micro-Doppler characteristics harder to see. The tail characteristics vary with range. Chips 3 and 10 are expanded in Figure 6.
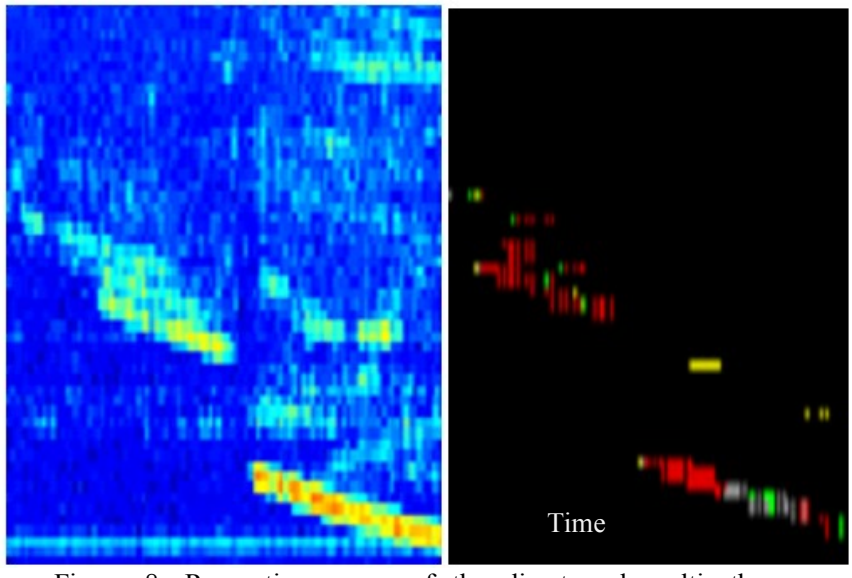

Figure 8. Range-time maps of the direct and multipath response from a human walking between and then emerging from between two metal and glass buildings (left), and the resulting tracks (right). This demonstrates the ability of a simple tracker in an urban environment, but fails to connect the two tracks of the same target when they move from the non-LOS to direct response.

\section{URBAN SURVEILlANCE MEASUREMENTS}

The tracker used a simple constant false alarm rate (CFAR) approach in order to evaluate how much more complicated an urban tracker should be. A diagram of the general measurement setup with the radar and the urban canyons is shown in Figure 3. The non-LOS tracking from a metal and glass building is on the right in Figure 8, with a human emerging from between the two buildings, shown in both the raw radar returns and the corresponding tracks. Individual chips out of range-Doppler maps used for the tracking are shown in Figure 7.

The direct and multipath response from a truck moving between two stucco buildings is in Figure 9. The multipath from a corrugated metal building is shown in Figure 10, with a human walking between and then emerging from between the two buildings. A similar measurement on a brick building is in Figure 11. The position and tracking of non-LOS subjects is affected strongly by the geometry of the radar in relation to the urban canyon as well as the motion of the subject.
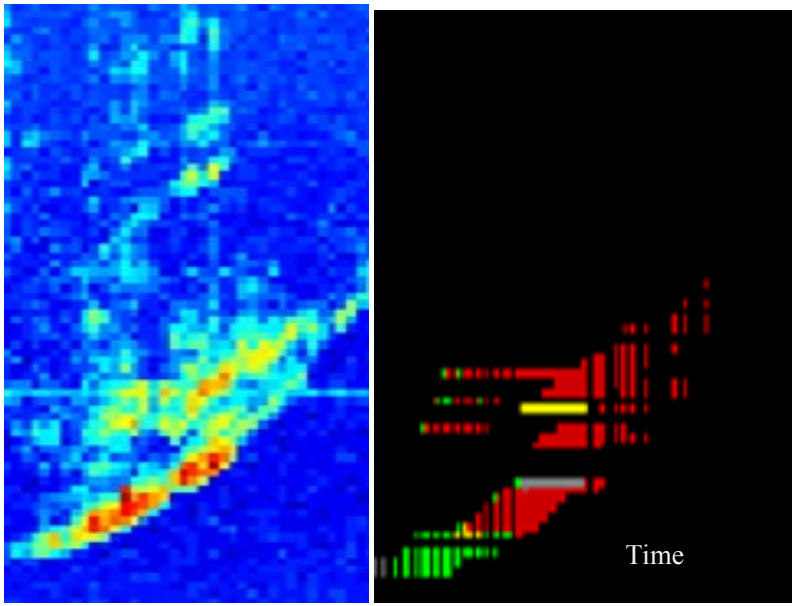

Figure 9. Range-time maps of the direct and multipath response from a truck driving between two metal and glass buildings (left), and the resulting tracks (right). Here there are both the direct and non-LOS detections that smear into one track.
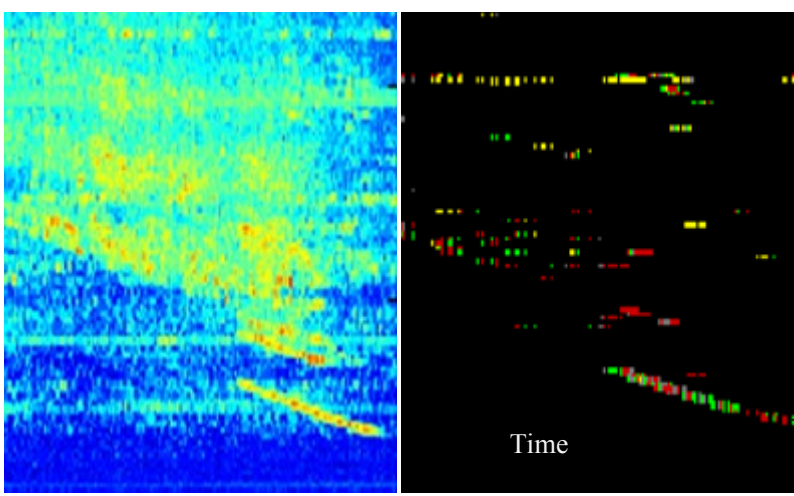

Figure 10. Range-time maps of the direct and multipath response from a human walking out from between two corrugated metal buildings (left), and the resulting tracks (right). Here the direct detections are tracked, but the corrugation scatters much of the radar energy making nonLOS tracking difficult. 
The variation in response due to the materials in the urban environment is apparent in the amplitude of the reflected signals. The urban canyons made of corrugated metal provided the most difficulty for tracking because of the large number of angles which could be reflected from the non-flat surface. Yet the tracker performed well on the high RCS vehicle, tracking its multipath, but less well on the lower RCS human when the dispersed energy lowers the non-LOS response to below detection thresholds. This is shown in Figure 10, which can be contrasted with Figure 8 or 9 .

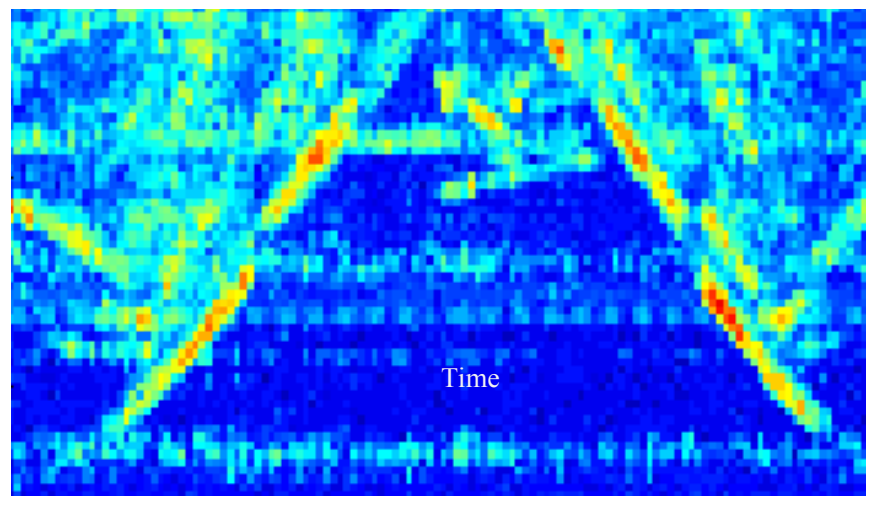

Figure 11. The direct and multipath response from a truck driving in and then emerging from between two brick and glass buildings. Here there are both the direct and non-LOS detections.

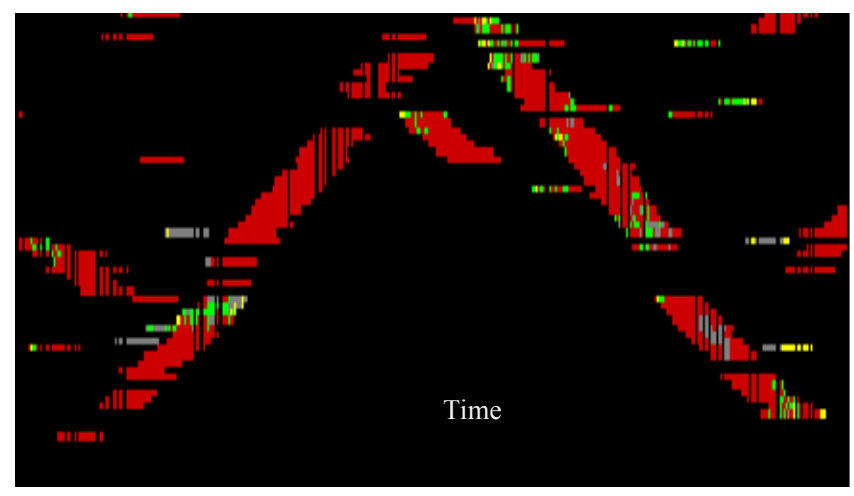

Figure 12. The tracks of a truck driving in and then emerging from between two brick and glass buildings. Here there are both the direct and non-LOS tracks which appear to smear together. Note that multiple detections can smear together. The tracks which appear to reverse direction are due to different reflections in addition to the urban canyon effects.

Comparison of the surface types in Figures $8,9,10$, and 11 shows the ability to track moving subjects, even as they go around a corner and enter an urban canyon. The data from Figure 8 shows a gap in the coverage as the subject is obscured by one building before it can get a two-bounce response from the building opposite, highlighting the importance of the geometry of the measurements. In contrast, Figures 9 and 10 show an overlap between the direct and multipath tracks in time, where the reflections are geometrically feasible before the vehicle has lost the direct path. This is due to a reduced angle for the observations into the urban canyon, as shown in Figure 4.
Figure 12 actually shows the vehicle moving in a different direction in the multipath track, then jumping in range to the direct track. Then change in observed velocity is not shown, but it crosses the zero-velocity line. Continuing a track from a reflected to a direct path appears challenging in this case because of the large discontinuous change in both range and Doppler; however, the presence of a continuing multipath return at a similar range to the old track and with a similar velocity to the new track serves as a potential link between the multipath and direct tracks.

In some cases, the tracking approach shows an inability to disambiguate two multipath tracks that are similar in range. This is especially clear in Figure 8, where the multipath tracks get combined into a single track due to the closeness of the target to the building. For urban environments when multipath tracks might be used for linking a subject to its previous multipath track, a more complex tracker is warranted.

\section{ClASSIFICATION IN URBAN ENVIRONMENTS}

Micro-Doppler signatures have been used as a biometric [14], and micro-Doppler features have been used in classification algorithms in $[14,15,16,17]$. MicroDoppler signatures and direction-of-arrival (DOA) estimates have been extracted at over nine meters range through a brick wall [18]. Fully polarimetric human radar signatures at different approach angles with respect to the radar have been collected [19]. Automatic target classification has also been done on data including multiple humans, wheeled vehicles, tracked vehicles, clutter, and animal classes [20]. Similar approaches were attempted with in an urban environment.

Subject classification in urban operations was found to be hindered by the two-way attenuation loss when the classification was heavily amplitude-based or required the detection of foot motion. An example of this is in Figure 7, where the direct path has significant micro-Doppler but the reflected paths were reduced in amplitude and lacking measurable micro-Doppler. The additional loss inhibits the measurement of lower-RCS body parts used in a microDoppler classification scheme, though the obscuration and partial reflections are a more severe source of loss. The lack of urban training cases for most radar classification systems means that approaches which are highly discriminative in a field may be less reliable in an urban operation.

\section{CONCLUSIONS}

Although much of the multipath response of a subject moving in urban environments is understood [21, 22, 23], urban canyons are less well studied. The detection and tracking of non-line-of-sight moving objects using multiple bounces was demonstrated across a variety of urban building materials with an urban radar surveillance system.

For utilizing multipath to see into the shadows of urban environments, VV polarization has the least loss, and $\mathrm{X}$ band was less susceptible to reflected loss than $\mathrm{Ku}$ or $\mathrm{Ka}-$ band systems for the cases we measured. Detection and tracking using multipath returns was demonstrated but 
there was no disambiguation of real or multipath sources. Some of the difficulties in tracking the subjects through their multipath reflections were shown, including large simultaneous discontinuities in both range and Doppler. However, using direct and related multipath returns could allow a tracker to follow by reducing the discontinuity to just Doppler and then just range.

Urban classification was shown to be possible but less predictable due to obscurations and signal attenuation. The uses of amplitude characteristics are especially difficult in an urban environment for classification unless the attenuation can be calibrated using a priori knowledge of the environment.

\section{REFERENCES}

[1] J. Durek, "Multipath exploitation radar industry day presentation," 2009.

[2] P. R. Barbosa, E. K. P. Chong, S. Suvarova, and B. Moran, "Multitarget-multisensor tracking in an urban environment: A closed-loop approach," in SPIE, vol. 6969, 2008.

[3] T. Trueblood, "Multipath exploitation radar for tracking in urban terrain," Master's thesis, Arizona State University, 2009.

[4] B. Krach and R. Weigel, "Markovian channel modeling for multipath mitigation in navigation receivers," in European Conference on Antennas and Propagation, March 2009, pp. 14411445.

[5] B. D. Rigling, "Urban RF multipath mitigation," IET Radar, Sonar and Navigation, vol. 2, pp. 419-425, December 2008.

[6] M. Mertens and M. Ulmke, "Precision GMTI tracking using road constraints with visibility information and a refined sensor model," in IEEE Radar Conference, May 2008, pp. 1-6.

[7] J. L. Krolik, J. Farrell, and A. Steinhardt, "Exploiting multipath propagation for GMTI in urban environments," in IEEE Conference on Radar, April 2006, pp. 65-68.

[8] Y. Bar-Shalom, Tracking and data association. Academic Press, 1987, pp. 157-190.

[9] Y. Bar-Shalom, F. Daum, and J. Huang, "The probabilistic data association filter," IEEE Control Systems Magazine, vol. 29, no. 6, pp. 82-100, Dec 2009.

[10] T. Kirubarajan and Y. Bar-Shalom, "Probabilistic data association techniques for target tracking in clutter," Proceedings of the IEEE, vol. 92, no. 3, pp. 536-557, Mar 2004.

[11] G. Pulford and R. Evans, "A multipath data association tracker for over-the-horizon radar," IEEE Transactions on Aerospace and Electronic Systems, vol. 34, no. 4, pp. 1165 -1183, Oct 1998.
[12] T. Sathyan, D. Humphrey, and M. Hedley, "Target tracking in multipath environments: An algorithm inspired by data association," in 12th International Conference on Information Fusion, 2009, pp. 1650-1657.

[13] T. Pizzillo, "Radio Frequency (RF) Measurements for Human Detection, Tracking, and Identification," ARL-TR-4302, September 2007.

[14] W.S. Marshall, J.L. Geisheimer, E.F. Greneker, D. Fry, R. Imamura, and B. Johnson, "Radar-based gait measurement for human identification," IEEE Auto ID 2002, Tarrytown, NY, March 2002.

[15] Y. Yang, J. Lei, W. Zhang, and C. Lu, "Target classification and pattern recognition using micro-Doppler radar signatures," Seventh ACIS International Conference on Software Engineering, Artificial Intelligence, Networking, and Parallel/Distributed Computing, 2006, pp. 213-217, June 2006.

[16] P. Kealey and M. Jahangir, "Advances in Doppler recognition for ground moving target indication," Proc. SPIE 6234, Automatic Target Recognition XVI, May 2006.

[17] M. Otero, "Application of a continuous wave radar for human gait recognition," Proc. SPIE 5809, Signal Processing, Sensor Fusion, and Target Recognition XIV, pp. 538-548, May 2005.

[18] A. Lin and H. Ling, "Through-wall measurements of a Doppler and direction-of-arrival (DDOA) radar for tracking indoor movers," IEEE Antennas Propagat. Soc. Int. Symp. Digest, vol. 3B, pp. 322325, July 2005.

[19] R. Tan and R. Bender, "Analysis of Doppler Measurements of People," Proc. SPIE 6239, Targets and Backgrounds XII: Characterization and Representation, edited by Wendell R. Watkins, Dieter Clement, 623908-1, 2006.

[20] I. Bilik, J. Tabrikian, and A. Cohen, "GMM-based target classification for ground surveillance Doppler radar," IEEE Transactions on Aerospace and Electronic Systems, vol. 42, No. 1, pp. 267-278, January 2006.

[21] R. Linnehan and J. Schindler, "Multistatic scattering from moving targets in multipath environments," in Proc. IEEE Radar Conf., Pasadena, CA, May 4-8, 2009, pp. 1-6, Paper 3215.

[22] R. Linnehan and J. Schindler, "Validating multipath responses of moving targets through urban environments," in Proc. IEEE Int. Radar Conf., Washington, DC, May 10-14, 2010, pp. 1036-1041.

[23] R. Linnehan and J. Schindler, "Multipath Analysis of Dismount Radar Responses," in Proc. IEEE Radar Conf., Kansas City, Mo, 2011. 